\title{
THE INFORMAL SETTLEMENTS OF LAHORE: UNDERSTANDING THE ROLE OF INFORMAL KATCHI ABADI IN THE CONTEXT OF AFFORDABLE HOUSING
}

\author{
Mehreen Mustafa*
}

\begin{abstract}
The city of Lahore is expanding at great pace in terms of its urban sprawl and demographics. The consequence of rural to urban migrations, as of arbitrary physical and social development in the city has triggered the process of urbanization at a rate which was never recorded before. Urban housing in this context is the most complex, in demand and unattended phenomena. The lack of efforts in bridging the gap between the demand and supply chain of urban housing in Lahore is promoting a culture of social and spatial inequity, and has produced a form of urban housing for the economically marginalized communities i.e. the Katchi Abadis.
\end{abstract}

In order to explore the above mentioned issues, a research project was initiated with the aim to understand the complexities of Katchi Abadis in Lahore, through the study of their existing realities, by employing tools of community based participatory methodology.

Based on the project conducted, the objective of the research paper was to explore Katchi Abadis as a form of affordable urban housing developed by the marginalized communities with limited resources and without financial or legal assistant of concerned authorities. The paper analysis the internal social and spatial politics of Katchi Abadis and their impact on the context and vice versa. The paper is aimed at highlighting the phenomenon of Katchi Abadis as an existing urban phenomena (neglected and segregated) which should be understood, in order to effectively cater to the growing housing demand in the city of Lahore.

Keywords: Katchi Abadi, informal settlement, urban housing, marginalized community, urbanization, affordable housing, community based participatory approach, Lahore.

\section{INTRODUCTION}

\subsection{Contextual Background}

Pakistan is one of the most urbanized countries in South Asia while its metropolitan city Lahore is the second highest as compared to other cities (Khaliq-Uz-Zaman, 2012). Studies have shown that despite physical congestion, lack of services and profound decrease in economic opportunities, the urban population in Pakistan, which constitute about thirty six percent of the total population, is expected to grow fifty percent of the total by 2030 (Nizami, 2010). Being a metropolitan city, Lahore is conceived as a city of economic and social opportunities thereby attracting huge influx of migrants from distant and nearby areas. This population shift, in terms of migration, has been translated into an increase in the urban population growth of Lahore and other major cities of Pakistan (Figure 1). Supporting the fact is a study which demonstrates that population growth in the city of Lahore alone has increased six times since 1951 (Pakistan Census report, 2002).

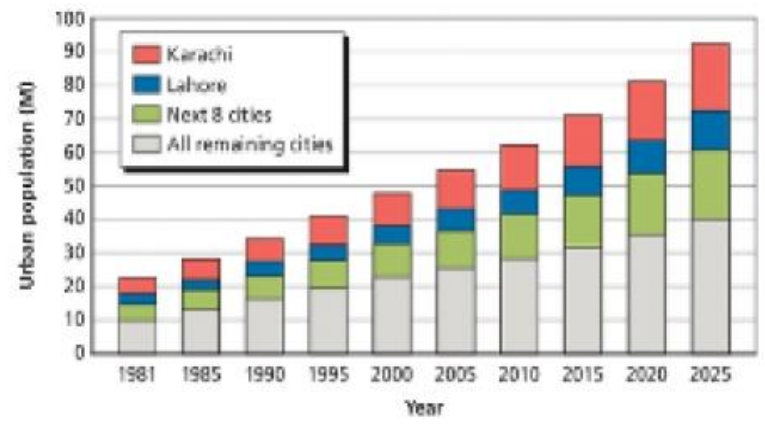

Figure 1: Population growth in Pakistan's Major cities (1981-2025) Source: Blank, et. al.,

* Mehreen Mustafa, Architect at Architorque, Assistant Professor, Institute for Art and Culture, Lahore Email Correspondance: mustafa.mehreen@gmail.com 
Like all the developing countries, the unprecedented urban growth in Lahore is caused by proliferating influx of migrants and has been associated with "pathological" growth of urban Katchi Abadis (Siddiqui, 1987). The demographic background of the migrants coming to the city of Lahore is very interesting, since the city not only attracts people from rural areas but also attracts large number of people from other relatively smaller cities. This specific type of migration is known as urbanization. Urbanization refers to the population shift from rural to urban residency, the gradual increase in the proportion of people living in urban areas, and the ways in which each society adapts to this change. The process of urbanization at one end has contributed in the city's economic growth by providing the required human resource. However, at the other end the process has also exerted great pressure on the city's limited and insufficient resources for its residents, especially in terms of housing. The housing crisis is not limited to the city of Lahore, but is a national issue as explained here:

"The seriousness of the housing issue in Pakistan can be judged from the fact that conservative estimates put the housing backlog at 9 million units which is increasing at 300,000 units annually because of unmet demand. 62 percent of this demand is for lower income groups" (Hassan, 2018: 32)

In Lahore specifically this housing crisis is cashed as an opportunity by real estate developers. The real estate developer sector of Lahore caters for the economically privileged residents/migrants announcing and developing attractive housing schemes, promising high quality of living and security. Agrarian land present around the periphery of Lahore (especially in the south) is being acquired at very nominal price and is sold to the privileged class at high price, by inculcating basic infrastructural facilities.

Nevertheless, the real estate sector is able to serve and make profit from relatively smaller section of the housing demand, catering only to the economically privileged class. More than seventy percent of the demand in terms of urban housing belonging to low or lower middle class remains unattended (Hassan, 1996). Due to the lack of affordable housing facilities, the marginalized section of Lahore has facilitied themselves in looking for options such as Katchi Abadis. The Punjab Katchi Abadis Act passed in 1992 defines the Katchi Abadis as "any area or part thereof which was occupied unauthorisedly before 31st December 2011 and continues to be so occupied and has at least fort dwelling units on it to be a Katchi Abadi". The definition of Katchi Abaids approved by the state, i.e. settlements on the land not owned by its occupants, segregates the residents, and the reason as to why these settlements began in the first place is not known (Mustafa and Mahmood, 2017).

The definition provided by the state also takes only "formal Katchi Abadis" in account, excluding the "informal Katchi Abadis" from the discussion and consideration.

Taking specifically the city of Lahore in account, two distinct forms of Katchi Abadis significant in their settlement pattern, physical morphology and social structure appear on the urban and social fabric. This paper refers to and defines these two distinct forms of Katchi Abaids as follows: Formal Katchi Abadis are not limited to but have the following characteristics:

\subsection{Formal Katchi Abadis}

a) Recognized by the state.

b) Permanent in its habitation and existence on specific piece of land.

c) High land use density.

d) Infrastructural facilities are in better shape than the other form of Katchi Abadis.

e) Usually occupied by refuges during or after 1947 partition.

f) Examples Hindu Camp, DAV Hostels: DAV hostels were first used as refugee camps during partition and now comprise of the third generation of refugees residing in extremely poor conditions (Mustafa et.al., 2016).

Table 1 and Figures 2 and 3 demonstrate the land use of the formal Katchi Abadi of DAV Hostels.

\begin{tabular}{|c|c|c|c|c|}
\hline S.NO & $\begin{array}{c}\text { Name of } \\
\text { Hostel }\end{array}$ & $\begin{array}{c}\text { Property of } \\
\text { Area }\end{array}$ & Area & Unit \\
\hline 01 & $\begin{array}{l}\text { D.A.V Hostel } \\
\text { number } 1 \\
\text { Court Street }\end{array}$ & SW-96-R-6 & $\begin{array}{l}23 \text { Kanal, } \\
15 \text { Marla }\end{array}$ & 206 \\
\hline 02 & $\begin{array}{l}\text { D.A.V Hostel } \\
\text { number } 2 \\
\text { Court Street }\end{array}$ & SW-96-R-6 & $\begin{array}{l}34 \text { Kanal, } \\
5 \text { Marla }\end{array}$ & 292 \\
\hline Source & Islam, Mus & abir, 2016 & & \\
\hline
\end{tabular}




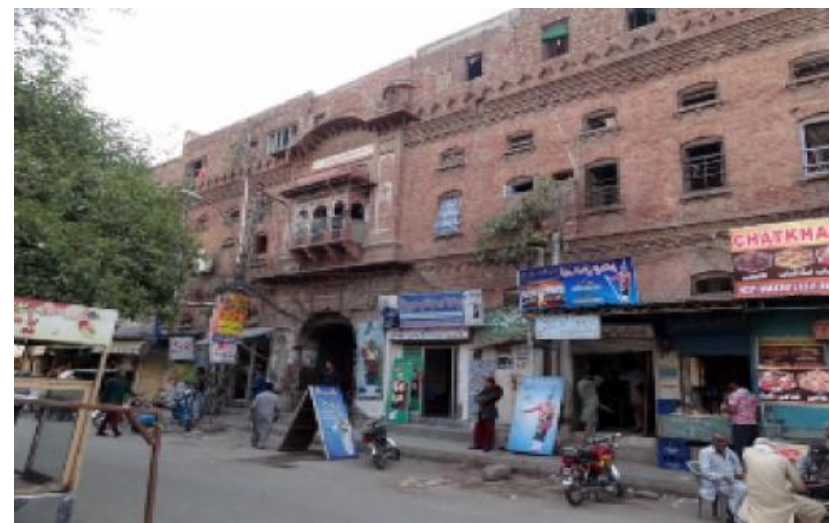

Figure 2: Front Façade of DAV Hostels

Source: Islam, Mustafa and Kabir, 2016

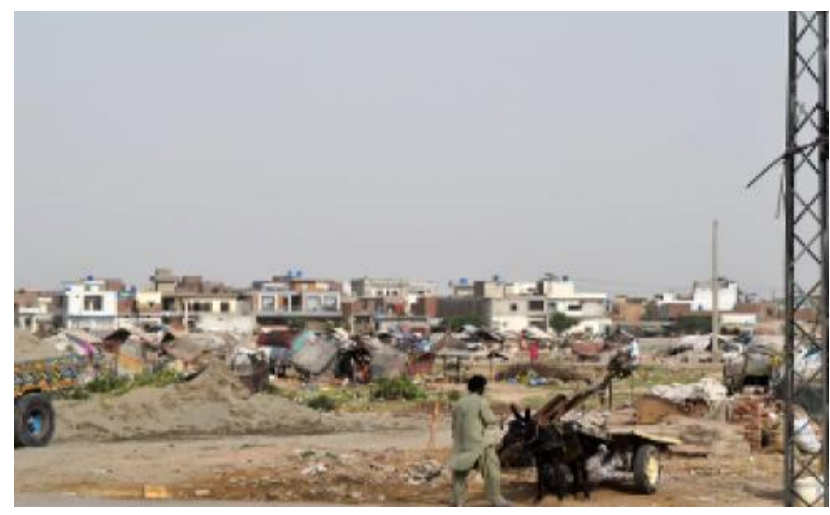

Figure 4: A view of the informal Katchi Abadi near Main Plaza Johar Town, Lahore

Source: Building Together, 2016

\subsection{Informal Katchi Abadis}

Informal Katchi Abadis also known as "Jhugiyan" are not limited to but have the following characteristics:

a) Unrecognized by the state.

b) Temporary in its habitation and existence on specific piece of land.

c) Low land use density.

d) Minimal or no infrastructural facilities available.

e) Occupied by nomads, gypsies, lower income section from rural areas and recently by Afghan refugees.

f) Example: Case study in consideration: Informal Katchi Abadis settlement near Mian Plaza in Johar Town, Lahore (Figures 4 and 5).

Although the two types of Katchi Abadis are distinct in physical and social form, yet they share the common issue of land ownership rights. Both types of Katchi Abadis are products of rapid urbanization and lack of affordable housing solutions through formal sector. This research revolves

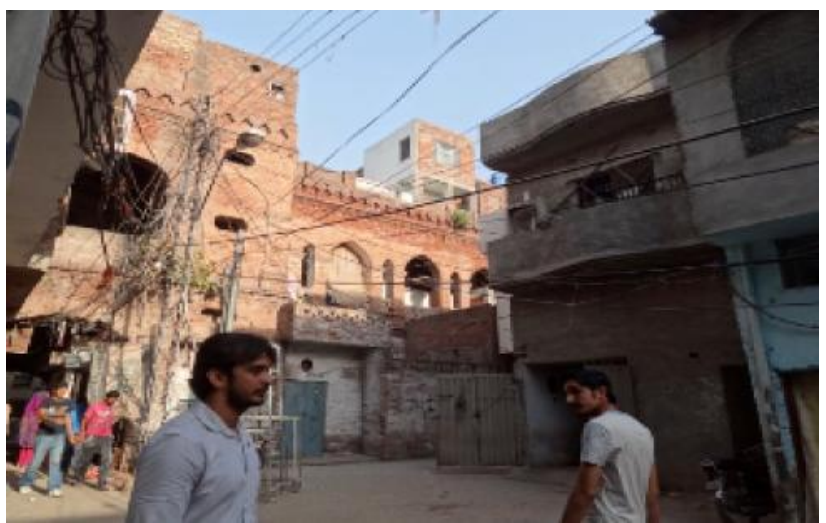

Figure 3: Expansion of DAV Hostels Source: Islam, Mustafa and Kabir, 2016

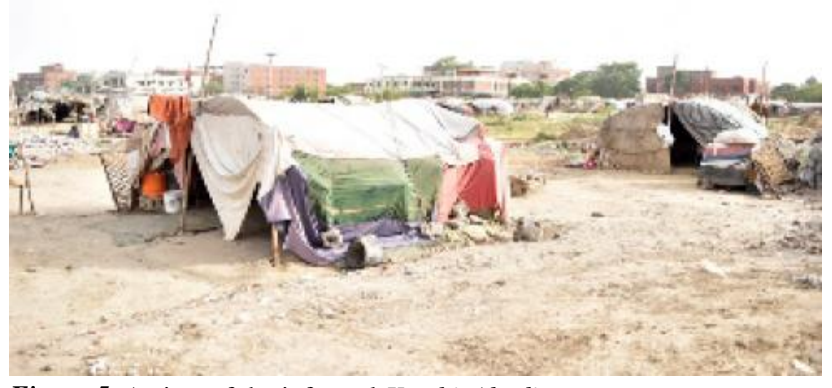

Figure 5: A view of the informal Ktachi Abadi near Main Plaza Johar Town, Lahore

Source: Building Together, 2016

around the most marginalized forms of Katchi Abadis i.e. informal Katchi Abadis or Jhugiyan.

\subsection{Problem Statement}

According to a report published by the World Bank, Pakistan is facing housing backlog of around nine million units and the number is increasing by 270,000 units every year (World Bank, 2016). Thus, fifty eight percent of the urban population of Pakistan lives in formal and informal Katchi Abadis and the percentage is increasing each year. Hassan, 1996 and Siddiqui, 1987 admit that in such a challenging urban context the informal Katchi Abadi settlements appear to be the only possible affordable housing opportunity for the low income groups, who migrate towards cities for better economic opportunities. Therefore, the informal settlements are indeed, despite of limited and poor resources, bridging the urban housing demand and supply gap.

There is no precise or widely accepted definition available which explains what exactly affordable housing is (Lin, 2011). 
However, a persistent and constant feature of low income community, as target groups of affordable housing, is present in all the definitions available.

Likewise a little has been studied and written regarding the characteristic and standards which define an affordable housing facility. Most studies in the field of affordable housing have been carried out to aid the state institutions, real state agencies or non-government organizations. What is missing in all such studies and work is the representation of the communities living in such settlements. Little or no work has been carried out exploring the concept of affordable housing with respecti to the perspective and narrative of people of living in low income settlements. Fichter and Turner (1972) have highlighted the dilemma of state of Non Govermental Organisation(NGO) funded affordable housing concepts, which lack epresentation and participation of low income communities.

Fichter and Turner's (1972) central thesis is that housing is best provided and managed by those who dwell in it. Keeping the autonomy and creativity of low income communities in mind, this paper, with the help of information collected through the study of concerned informal Katchi Abadis, establishes how the settlement in question can be explored as a form of affordable housing designed by low income communities, so that the quality of construction improves and thus the livilihoods are impacted positively. Although the informal Katchi Abadis of Lahore house the most marginalized community of the society, yet these settlements are part of a very complex socio-cultural system that cannot be worked from a distance (Mustafa and Mahmood, 2017). Contrary to common conception, where these settlements are avoided and hid as being the ugly face of the city, these informal Katchi Abadis can be seen as creative solutions devised by the so called "uncreative" and "uneducated" section of the society, as adaptations of their version of metropolitan life. These settlements can be seen as the only workable dwelling for low-income groups, when there are no legitimate models of affordable housing available for them (Eezdi, 2016).

\subsection{Aims and Objectives}

Thus the objective of this study is as follows:

- To understand the complex phenomena of informal Katchi Abadis

- To explore the informal Katchi Abadis of Lahore as a form of affordable housing solutions designed by the concerned marginalized communities.
- To explore the informal Katchi Abadis as a workable solutions in the field of urban housing, rather than an urban eye sore.

- To study and document the built and social environment of an informal Katchi Abadi in order to understand the internal dynamics and achieve an in-depth insight of communities life.

- To study and document the impact of the informal settlement on its immediate context and vice versa.

\subsection{Research Questions}

The paper revolves around the following main research questions:

- Can the informal Katchi Abadi be explored as form of affordable urban housing developed by the marginalized community with limited resources without financial or legal assistant of concerned authorities?

- Are informal Katchi Abadis bridging the demand and supply gap of housing?

- Can the above mentioned questions be explored by analyzing the internal social and spatial politics of Katchi Abadis and their impact on the context and vice versa?

\section{SOURCES AND METHODS}

The basic information and data required for the research issue was generated with the help of following two sources:

- Literature review of publications and other relevant material available on the subject.

- Maps, demographics, photographs and interview data generated from three day workshop titled "Building Together: Learning and designing with the Katchi Abadis of Lahore" (details below).

Building Together: Learning and building with Katchi Abadi was a three day workshop initiated by the Community based Participatory Learning and Design Center, in Lahore with the intension to build valuable links with the Katchi Abadi Community. The project was conducted in the form of a workshop, and focused on exploring and understanding the notion of community based participatory learning and design, by taking an informal Katchi Abadi site located near Allah Hu Chowk, Johar Town, Lahore (Figures 6 and 7).

The idea of community based participatory approach was an integral part in the conception, development and conduction process of the workshop. Community based 


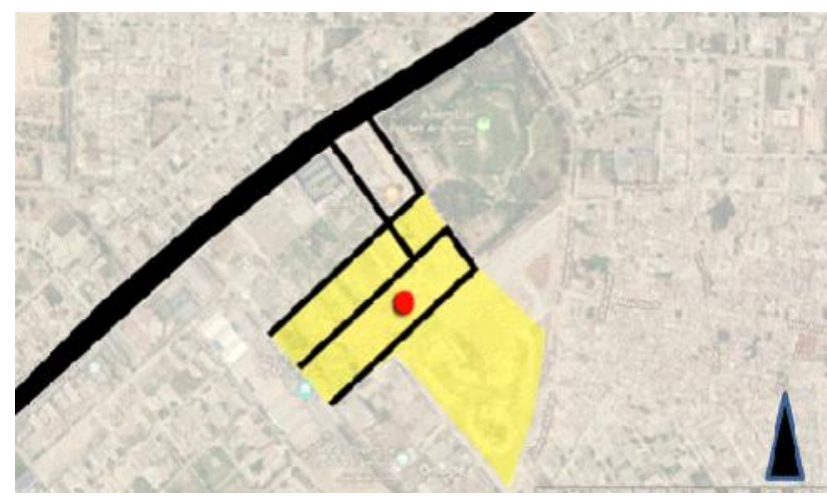

Figure 6: Site Map of the Informal Katchi Abadi near Mian Mir Plaza, Lahore.

participatory learning, research and design is a collaborative and multi-disciplinary field, which places the community at the center of the design process to yield creative and sustainable design solutions. The political dimension of user empowerment and democratization is essential to participatory approach, where the community is viewed and engaged as an active participant in the process of development. Therefore, following the guidelines, the key participants of the workshop i.e. the community and the students were placed at the center of the research and were actively involved in the decision and development of the workshop through meetings and discussion sessions.

The participants were first introduced to the idea of community based participatory learning, research and design. Various concepts, approaches and methodologies being designed and practiced all over the world were also discussed. Debates such as politics of informal settlements, ethics, tools and methods of field research, site dynamics and workshop briefing was inculcated in the workshop, with the help of presentations, interactive dialogue sessions and Ted Talk screenings.

With the basic understanding of the ethics, tools and methods of participatory research and mapping, the participants were introduced to the approved site i.e. the informal settlement near Allah Hu Chowk in Lahore. The participants (students and community) mapped and documented the location, number, land use, typology and characteristic (in terms of materials, interiors, shapes and layout) of various community structures with the help of questionairres and forms. The context, facilities, natural elements and basic information regarding the economic and social backgrounds of the community members were also mapped, in order to understand the impact and relation of social and built environment on each other. At the end of the second day of the workshop, a community meeting was arranged, where

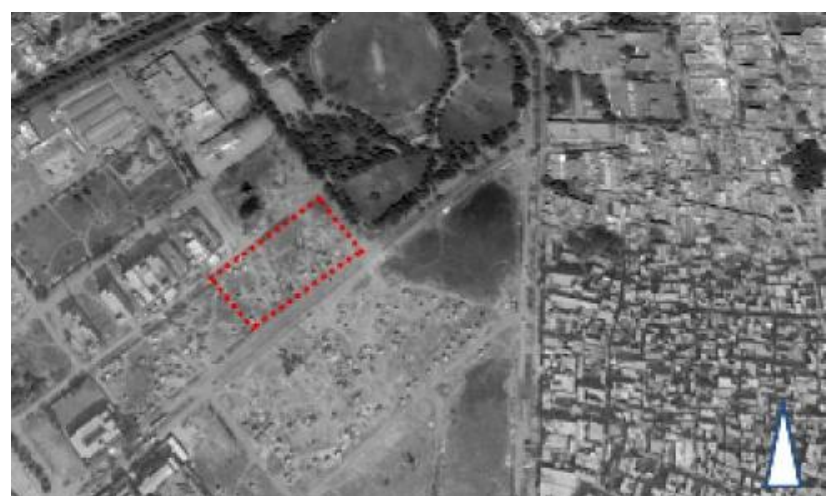

Figure 7: The area within the informal Katchi Abadi which was mapped during the workshop

the students and the community members discussed and analyzed the research conducted.

\section{ANALYSIS AND DISCUSSION}

The Katchi Abadi's, which are present in almost all the major cities of Pakistan, are an important part of the built environment which help shape them as well, yet the users of the city never acknowledge their presence. These settlements are also neglected during the formal city planning processes. The communities generally, and the community of the concerned Katchi Abadi particularly, may appear as homogeneous entity bounded primarily by economic interest, whereas in reality the concerned community is not only heterogeneous but contradictory as well. The multiple identities and their overlapping often generate common identity and narrative, allowing the conflicting heterogeneity present in the community to settle and exist peacefully. The concerned community of Katchi Abadi likewise, is composed of different communities, of distinct caste, sect and economic backgrounds.

The site in question is situated near Allah Ho Chowk, Johar Town, is mostly surrounded by commercial, educational, health and recreational land uses (Figures 8 and 9)

Most of the informal Katchi Abadis in Lahore (inclusive of the concerned one) are facilitated by four major sources:

a) The Lahore Development Authority (LDA) or other relevant concerned authorities, that house migrants on the land which is either property of the government but prone to influences of land mafia or on land with disputed ownership. This explains to great detail why informal Katchi Abadis are usually developed or found on a large piece of land surrounded by potent commercial context in urban center or peripheries. 


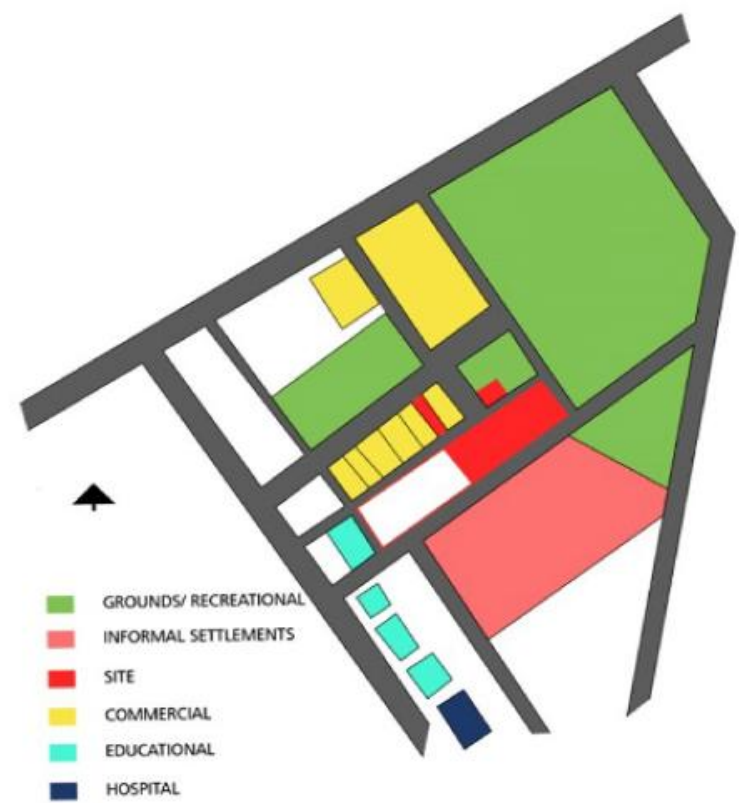

Figure 8: The area within the informal Katchi Abadi which was mapped during the workshop

b) The land mafia also houses the migrants on disputed land. The migrants find a temporary shelter or space to live in, where as, the land mafia does not have to employ paid workers to guard the land they intend to grab. Eventually the residents are either evicted or re settled to another disputed land, also managed by the land mafia, once the mafia acquires the permission to develop or sell the land.

c) A relatively small section of the residents are also facilitated by their employers who are living / working near the informal settlements, in collaboration with the main facilitator i.e. the land mafia or government authority.

d) A number of residents are also facilitated by their relatives who are already living in the informal Katchi Abadis as residents. This type of facilitation usually takes place when either a family is evicted from another informal Katchi Abadi or has recently migrated to the city. This particular type of facilitation also indicates the presence of a complex network between various informal Katchi Abadis facilitated by the four major sources explained.

The physical form and social structure of the informal Katchi Aabdis due to their temporal and adaptable trait therefore demonstrates a non-homogeneous settlement pattern. The

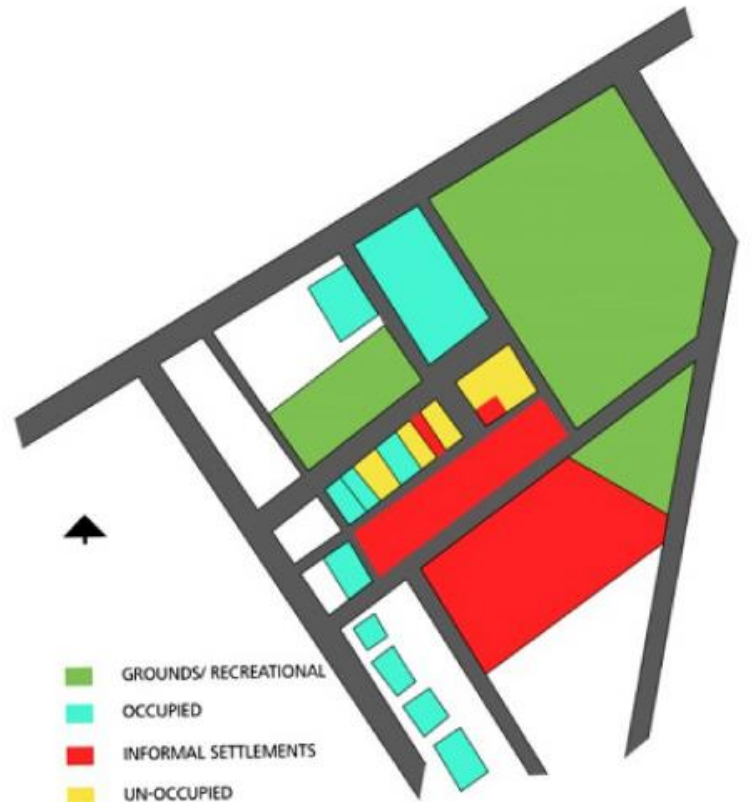

Figure 9: The area within the informal Katchi Abadi which was mapped during the workshop

settlement pattern is dictated by the demands of the facilitator and the land on which they are formed. The concerne $d$ Katchi Abadi at Mian Plaza has existed since 2004 (Figures 10,11 and 12) whereas different settlers have occupied the Abadi and various portions have been evicted over the years. Figures 10,11 and 12 also illustrate the impact of development projects on the urban morphology of the site in discussion and vice verse.

The process of selected eviction or re settlement pattern is guided by land potential and social structure of the informal Katchi Abadi community, in terms of their ethnic backgrounds, caste and religious believes. Likewise the apparently monotonous form of informal settlement in consideration has also organized itself (spatially and socially) with respect to ethnic backgrounds and caste. Therefore the spatial organization of the informal Katchi Aabdi in question is divided into (physically unmarked) different zones or areas belonging to a specific caste or ethnic group (Figure13).

The eviction or re settlement process of the residents from a specific portion therefore only disturbs the social and spatial autonomy of specific ethnic groups of the settlement. The facilitator in charge and the ethnic background of the residents are also directly associated with livelihoods, culture and social behavior of the residents. As can be seen in Table 

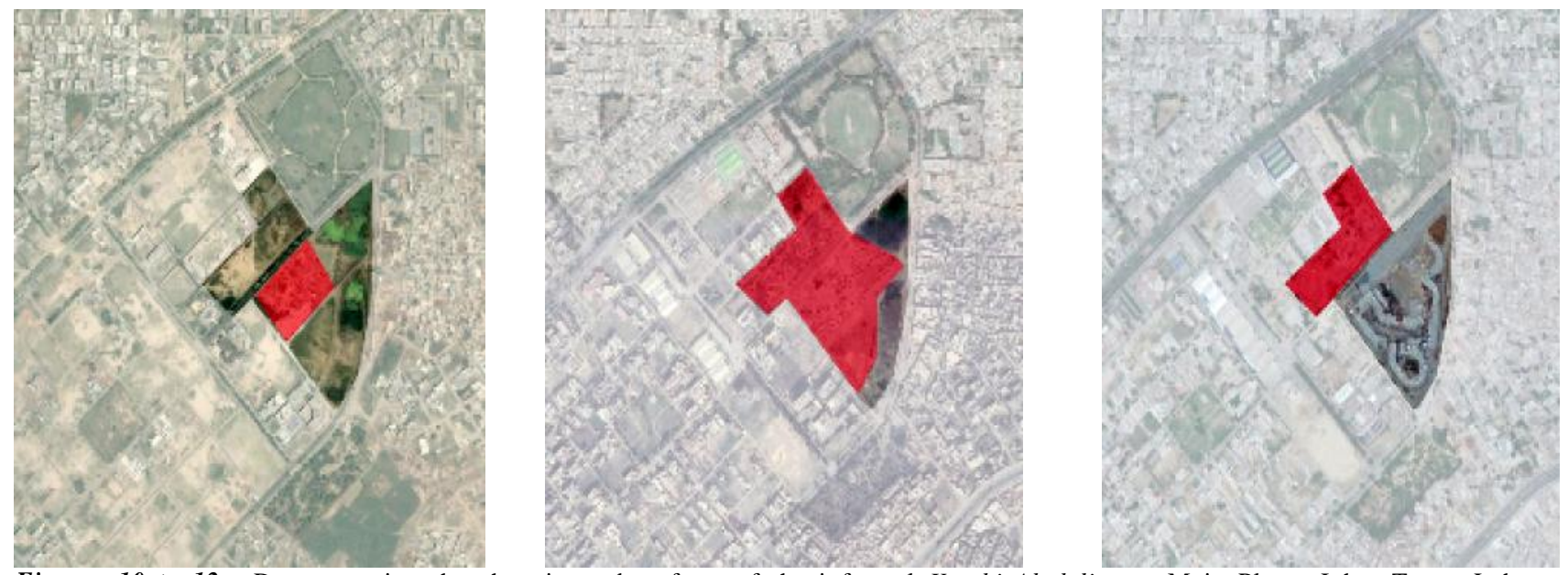

Figures 10 to 12: Demonstrating the changing urban form of the informal Katchi Ababdi near Main Plaza, Johar Town, Lahore. Source: Google earth , 2004, 2016 \& 2018

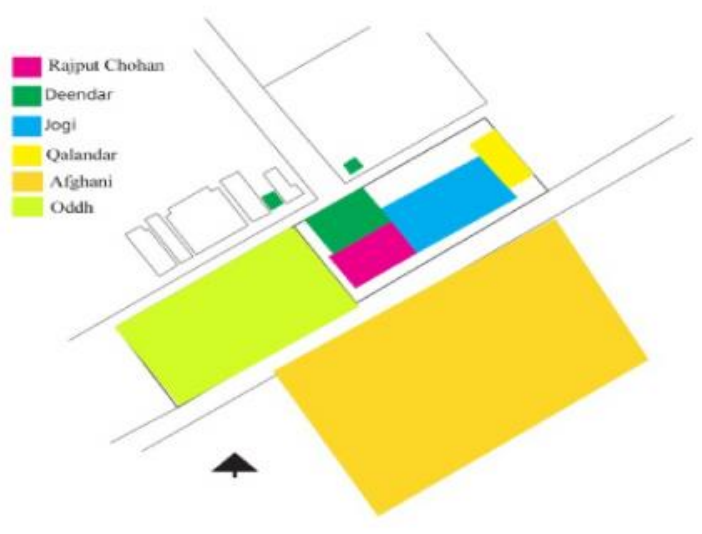

Figure 13: The spatial organization of the settlement with respect to caste and ethnic background

Source: Building Together, 2016

2, the community of the informal Katchi Abadi in consideration is majorly composed of Rajput Chohan, Deendar, Qalandar, Jogi, Oddh and Afghanis. They earn their livelihood by selling toy bangles, rings etc. to charming snakes, selling milk and working in nearby vicinity as maids and watchmen. Their belief system and means of earning livelihoods are directly reflective in their way of living. Oddh and Deendar communities are economically privileged and religious conservatives.

Special built form arrangements facilitating communal living are seen in the locality, for instance spaces are designed

\begin{tabular}{|c|l|l|}
\hline \multicolumn{3}{|c|}{ Table 2: Details of D.A.V Hostels. Hindu Camp - Lahore } \\
\hline S. No & $\begin{array}{c}\text { Ethnic Ggroup } / \\
\text { Caste }\end{array}$ & \multicolumn{1}{|c|}{$\begin{array}{c}\text { Means of Earning } \\
\text { Livelihood }\end{array}$} \\
\hline 01 & Rajput Chohan & Maids \& Watchman \\
\hline 02 & Deendar & $\begin{array}{l}\text { Maids, watchman \& } \\
\text { rikshaw drivers }\end{array}$ \\
\hline 03 & Odhh & Livestock \\
\hline 04 & Jogi & Snake charmers \\
\hline 05 & Qalandar & $\begin{array}{l}\text { Selling rings , Bangles \& } \\
\text { Toys }\end{array}$ \\
\hline 06 & Afghani & $\begin{array}{l}\text { Collecting and selling } \\
\text { garbage }\end{array}$ \\
\hline Source: Building Together, 2016 \\
\hline
\end{tabular}

such that children can engage in religious education while women study in pardah (segregation). Therefore the homes of Oddh and Deendar communities are covered from all sides by either walls made of thatch and bamboo, or with the help of a piece of cloth. The other community members, except Afghanis, have designed their homes in a way that they can lift or drop the walls (made of pieces of cloth) of their homes depending on the weather conditions (Figure 14). Unlike Deendar, Oddh, Rajput Chohan and Afghanis, the Jogi and Qalandar communities celebrate their religious practices by building a small decorative platform inclusive of alum and space for diya near their homes (Figure 15). 


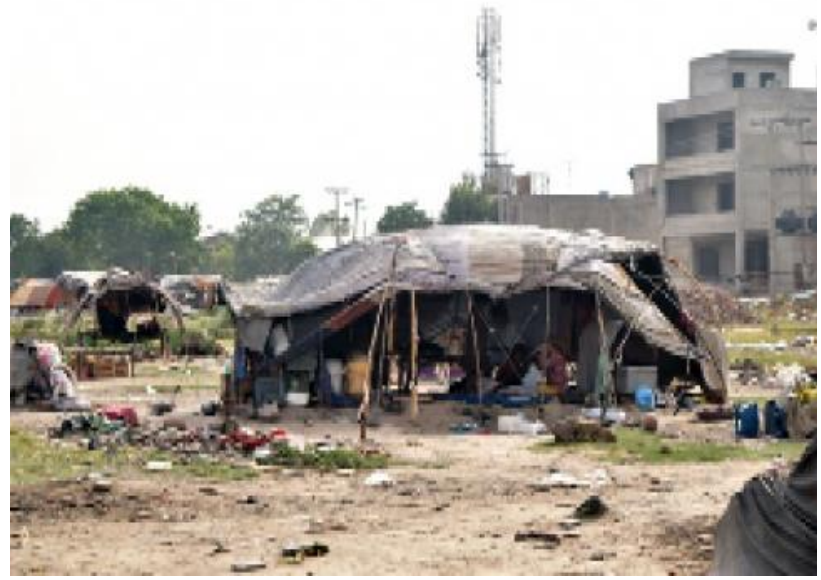

Figure 14: The walls are covered using curtains made from piece, of cloth and are dropped or lifted as per weather conditions.

Source: Building Together, 2016

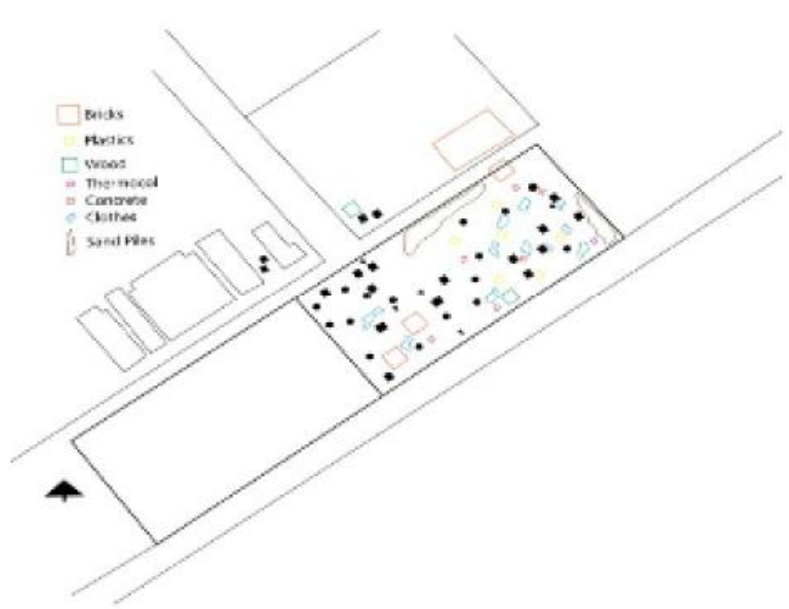

Figure 16: A number of resource materials found on the site. Most of it was used by the inhabitants to repair their dwellings and for other purposes as well.

Source: Building Together, 2016

\section{FINDING AND ANALYSIS}

\section{Low Cost and Sustainable Construction Techniques and Material:}

Apart from bamboo and thatch roof, almost all the material used in the construction of the Jhugis is picked from trash and then recycled. The settlement itself is full of material used in the construction of Jhugis for example mud, bricks, ropes, stones, pieces of cloths and plastic (Figures 16). Reducing the cost of material to significant level is essential, irrespective of the fact that the size and design of the Jhugis is not uniform. Jhugis are built in various shapes and sizes, keeping the family size and demand of the dwellers in mind

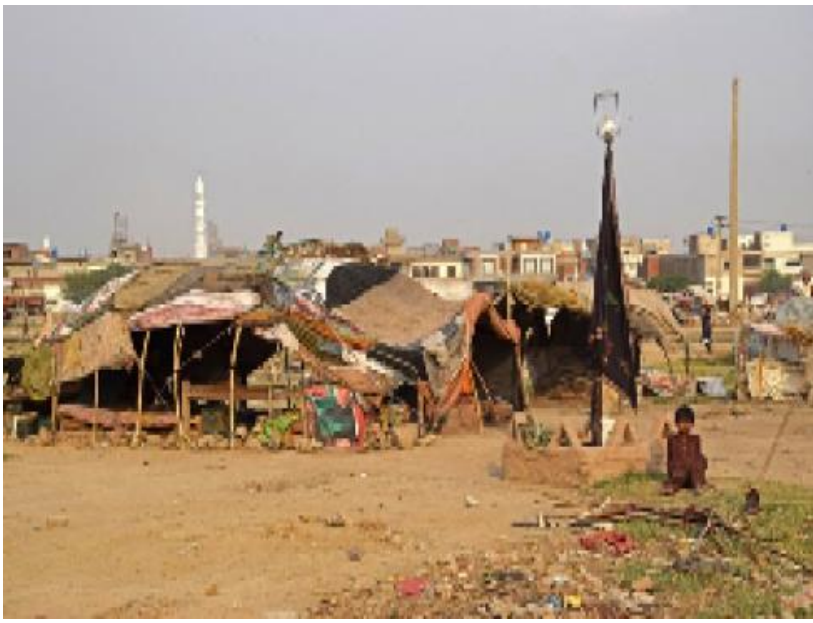

Figure 15: A small platform, clusive of alum, is made using bricks and mud, to commemorate religious beliefs.

Source: Building Together, 2016

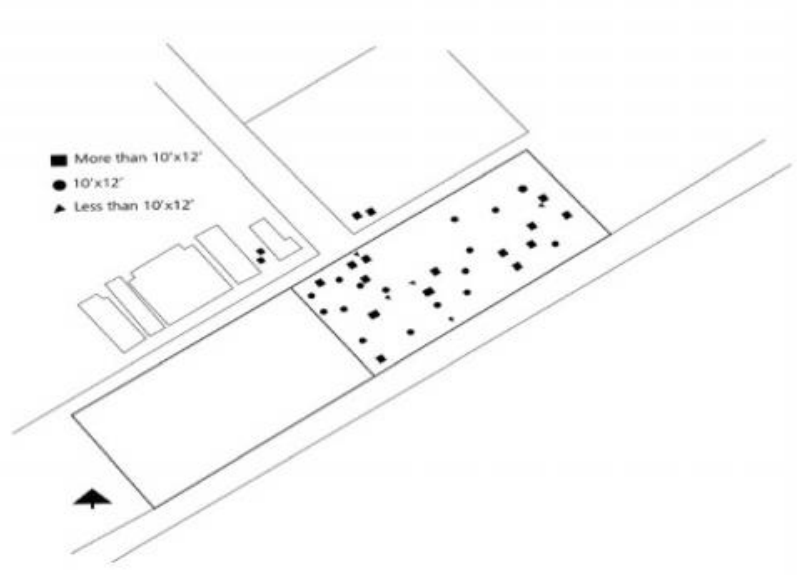

Figure 17: A number of different sizes of huts were seen on the site. The different size usually depended on the number of family members and their needs.

Source: Building Together, 2016

(Figures 17). An interesting community based approach is employed for the construction of Jhugis, where the human resource in terms of labor is not a paid one. People or neighbors from close proximity volunteer themselves as unpaid labor for the construction process. The vernacular knowledge of building a Jhugi is inherited and even a twelve year old child is aware of the construction techniques. These people have the knowledge of exact number of structural supports and systems required for a specific type of Jhugis to withstand the natural and manmade forces (Figures 18 and 19). 


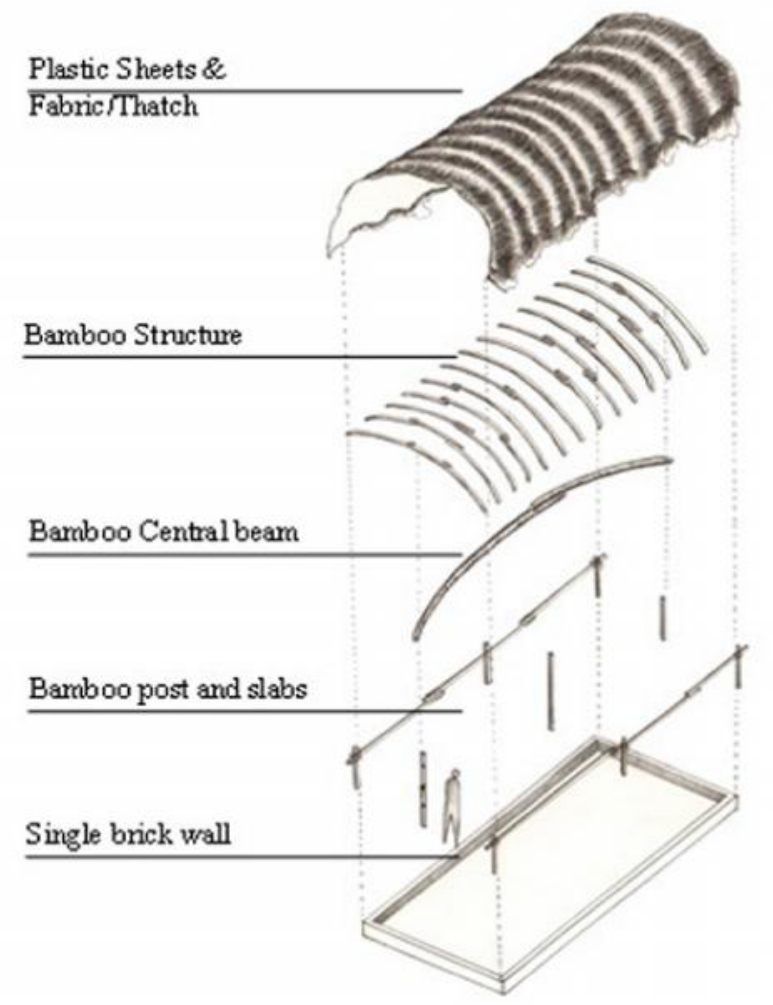

Figure 18: Axonometric view of the Jhugi design (Type A) Source: Building Together, 2016

\section{Relying on Minimum or Existing Infrastructure}

The facilitator of the concerned informal settlements range from government officials to influential land grabbers. The settlers are employed by their facilitators, as a cost effective tool to maintain and hold on to a piece of land. Therefore, the provision of services like electricity, water supply and sewerage, are highly discouraged. The community, even with the help of their own resources, cannot install or use an energy source without the permission or consent of their facilitator. The facilitator, most likely always, denies any prospect which helps the community to get comfortable on the land. Getting comfortable means inviting any unpleasant resistance during eviction or resettlement.

The community concerned, however had devised creative strategies to cater and fulfill their energy and mobilization demands. A basic network of road was made available around the periphery of the site. The settlers, with the help of waste material available on site, such as sand, stones, brick, gravel and mud built pathways and unpaved roads up to three to five feet wide, to shorten their commute (Figures 20)

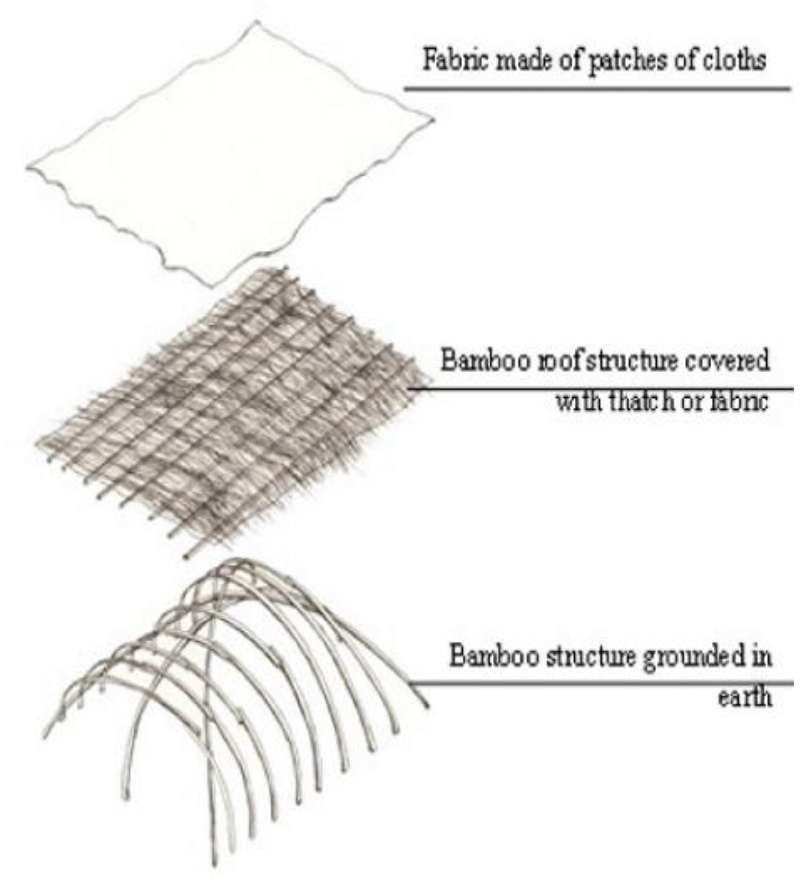

Figure 19: Axonometric view of the Jhugi design (Type A) Source: Building Together, 2016

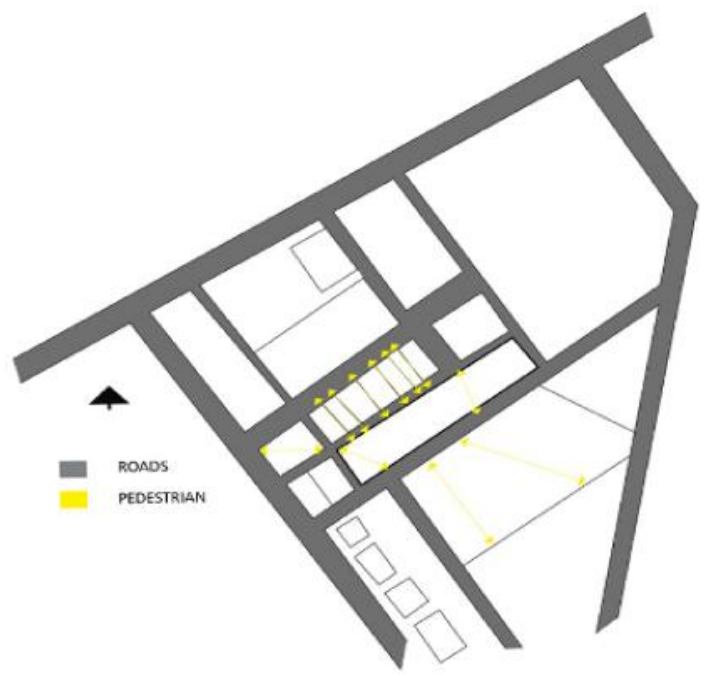

Figure 20: The road network around the site is well maintained. A few informal pedestrian pathways can be seen around the site, which were made as shortcuts, cutting thorough empty plots. Source: Building Together, 2016 


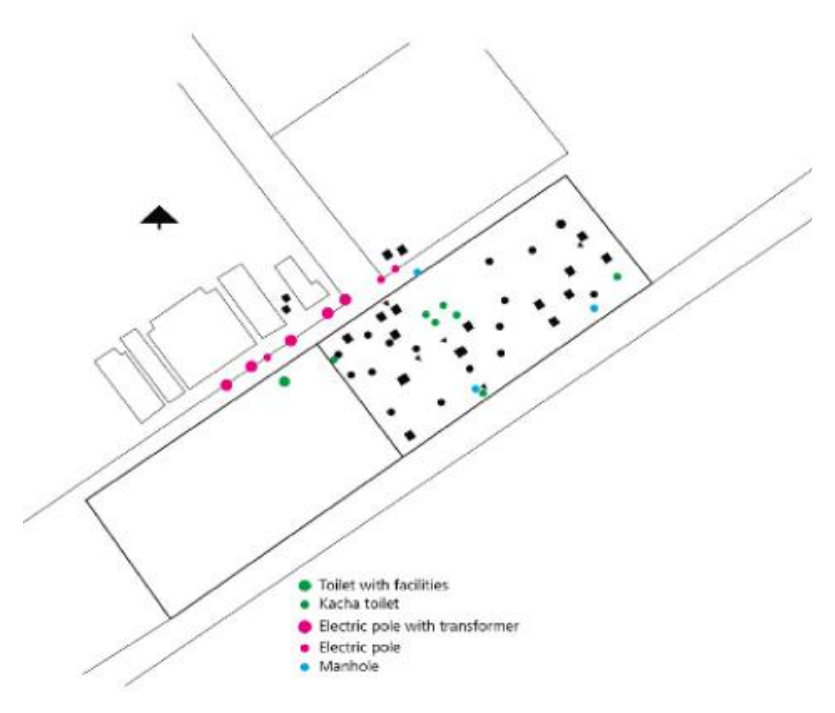

Figure 21: Even though a number of infrastructural elements were present on the site but the inhabitants were not allowed to use the facilities. For example, a number of electrical poles were present on the site but majority of the huts are without electricity.

Source: Building Together, 2016

Few Jhugiyan were also privileged enough to borrow electricity from existing light poles or nearby plazas. Others relied on solar panels for their energy consumption. Whereas most of the community depended on day light and oil lamps to meet their respective energy demand (Figures 21). Water was fetched in fixed hours from a source provided by the facilitator. The settlers had to pay for it some times but most of the time it was free of cost. Furthermore, interesting amalgamation of below mentioned design strategies were practiced by different groups of people living in the settlement, for solid waste management (Figure 22).

- A group of houses had rented out a toilet in nearby plaza.

- Other group of houses had installed a katcha toilet, comprising of walls made of fabric and bamboo, on existing manholes.

- Another group of houses had dug a pit and built katcha toilet around it. These pits were either cleaned or the toilet would eventually move to new location. Depending upon the need and limited resources, every household had devised creative strategies to meet their energy and other daily demands, without exerting additional pressure on the available resources.

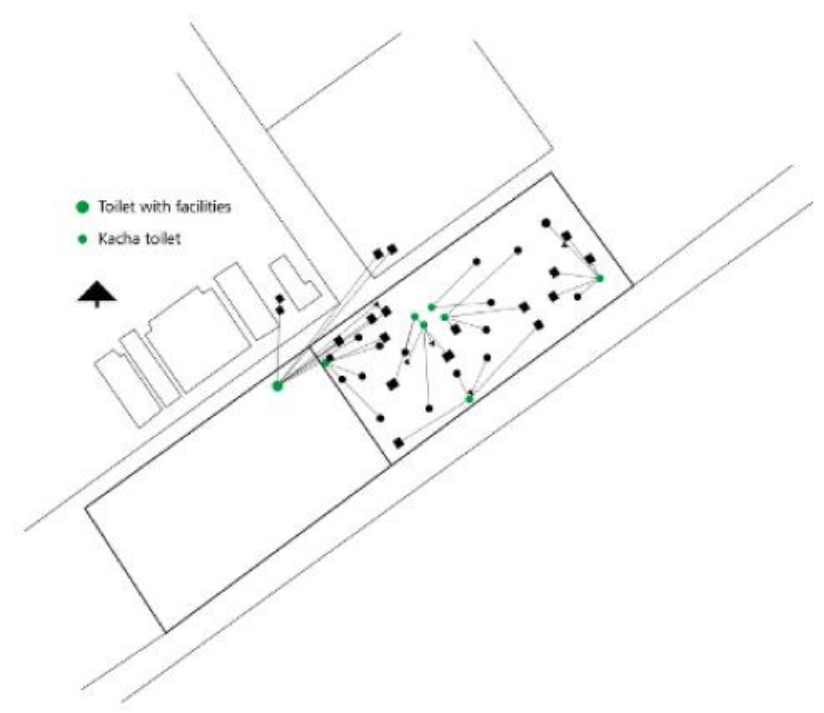

Figure 22: A number of Kacha toilets were found on the site, which were being used by the inhabitants. The toilet with facilities was to be paid for and only those who could afford it, were using it. Source: Building Together, 2016

\section{- Temporal and adaptable}

Embedded in the organizational structure of the concerned informal Katchi Abadi is the trait of being a temporal and adaptable settlement. Nothing in the informal Katchi Abadi is fixed, be it its demographics or physical form, everything can adapt and change according to the demand of time and land. The integral characteristic of being temporal and adaptable settlement always works in favor of the informal Katchi Abadi residents, the settlement itself and the city.

Since these settlements provide services, such as human resources ( especially for domestic chores), dairy products (especially milk) and venders, serving the formal as well as informal economy of the city. Temporal and adaptable is the only trait of the informal Katchi Abaids which also acts as a challenge while the upgradation is in consideration, since the settlement is in a state of transition.

The informal Katchi Abadis have addressed the demand and supply gap of urban housing crisis since many years, but are always viewed as problems rather than as an opportunity. The phenomena of the informal settlements in the southern hemisphere have proven to be inevitable. 
It is high time that these settlements are explored as parallel housing phenomena's rather than eye sores. As explained by Turner (1969) there is a need to take view of these informal settlements as highly successful solutions to housing problems in urban areas of the developing countries and work towards supporting them through technical assistance, access to credit and managerial advice.

\section{ACKNOWLEDGEMENTS}

- Participants of Building Together: Learning and Designing with the Katchi Abadis of Lahore.
- Ibrahim Mahmood, Alina Sohail, Aliya Khalid, Asim Mehmood, Hafiz Zain, Haleema Bhatti, Hamna Ahmed, Abdul Haseeb, Kamran Jameel, Maria Afzal, Munzza Tanveer, Waqas Shabir, Sadia Riaz, Syed Ahmed ujtaba, Nadir Hayat Khan, Shaan Akram, Allah Rakha Tufail, M Afzal Tufail \& Umer Arif.

- Society for Cultural Education and Institute for Art and Culture.

- Center for community Based Participatory Learning and Design.

\section{REFERENCES}

Blank, C, Jonah, et. al., "Drivers of Long-Term Insecurity and Instability in Pakistan Urbanization", RAND Corporation, California.

Eezdi, R, "Katchi Abadis: The Backstage of the City - TNS - The News on Sunday", The News on Sunday, viewed 24 Nov. 2016, from tns.thenews.com.pk/katchi-abadi-in-the-backstage-of-city/\#.XMLqwbdKjIU.

Fichter, R, and J. Turner J.F.C., 1972, Freedom to Build, NewYork MacMillan Publishing Company.

Hassan, A, 1996, "National Conference on Business Administration and Economics", The Role of the Informal Sector in Provision of Urban Housing and Facilities, viewed 10-06-18, from settlements/land-housing/the-role-of-the-informalsector-in-provision-of-urban -housing-and-facilities.

Hassan, A, 2018, “Houses Or Housing?”, viewed 3 Oct. 2018, from arifhasan.org/human-settlements/houses-or-housing.

Lin, J, 2011 “The Development of Affordable Housing: A Case Study in Guangzhou City, China.” Department of Real Estate and Construction at Kungliga Tekniska Högskolan, Royal Institute of Technology.

Mustafa, M, and Ibrahim M., 2017, “A Walk in the Dust”, THAAP Journal 2017: People and the City, 2017: $225-232$

Mustafa, M, et. al., "The Isolated Communities of South Asia: An Outcome of the Political Mapping from the Indian Subcontinent”, THAAP Journal 2016: People's History of Pakistan, 2016: 267-284.

Nizami, 2010 "Labour Force and Employment", Econ Surv, viewed 6-7-18, from http:// www. finance.gov.pk/survey/chapter_10/16_Population.pdf.

Pakistan Census Report, 2002 Pakistan Population 2019 (Demographics, Maps, Graphs),viewed 6-10-18, from worldspopulationview.com/countries/pakstan-population/.

Siddiqui , T., Ahmed., 1987, "Shelter For The Urban Poor: An Experiment By Hyderabad Development Authority", Hyderabad Development Authority, Hyderabad.

Turner, J.F C, 1969, Housing by People: Towards Autonomy in Building Environments, Marion Boyars, UK.

World Bank, 2016, "Project Information Document/Integrated Safeguards Data Sheet (PID/ISDS)",World Bank, Report No: PIDISDSC18646, [Online].

Zaman, Khaliq Uz. 2012 "Urbanization of Arable Land in Lahore City in Pakistan; A Case-Study." Europen Journal of Sustainable Development, Vol. 1: 69-83. 